\title{
Developments in the meiotic analysis of hybrids. V. Second-order models for tetraploids and pentaploids
}

\author{
C. G. D. CHAPMAN \& G. KIMBER* \\ PBI Cambridge, Maris Lane, Trumpington, Cambridge CB2 2LQ, U.K. and *Department of Agronomy, Curtis Hall, \\ University of Missouri- Columbia, Columbia, MO 65211, U.S.A.
}

\begin{abstract}
'Second-order' models for meiotic analysis have been developed for tetraploid and pentaploid hybrids to address a number of situations intermediate between the established 'first-order' models. They all incorporate a third parameter of relative affinity, $z$, intermediate between $x$ and $y$, the relative affinities of the most and least closely related genomes respectively. Four such models are described: a $2:(1: 1)$ and a $(2: 1): 1$ for tetraploid hybrids; and a $2:(1: 1): 1$ and a $(2: 1): 1: 1$ for pentaploid hybrids. Examples of their application are given. Where $z \approx x$, or $z \approx y$, then interpretation through the simpler first-order models is appropriate. In other cases application of second-order models can reveal more subtle relationships among the genomes in a hybrid.
\end{abstract}

Keywords: meiotic models, genome analysis, pentaploids, tetraploids, Triticum timopheevii.

\section{Introduction}

The meiotic analysis of polyploid hybrids through the application of mathematical models of chromosome pairing offers a more objective way to assess genome, and hence species, relationships than does simple inspection of meiotic figure numbers. The models hitherto described in this series of papers (Chapman \& Kimber $1992 \mathrm{a}-\mathrm{d}$ ) relate meiotic behaviour to two parameters $x$ and $y$, which are the relative affinities between the more and less closely related genomes in a hybrid. Since, by definition, $x+y=1$ in practice the value of only a single independent variable is sought.

More complex models developed by Crane and Sleper (1989a and b) for triploid and tetraploid hybrids allow more variables that describe meiotic behaviour to be estimated. In fact these models are 'overparametized', as they handle more variables than there are degrees of freedom in the datasets being analysed. In consequence very good agreement can be obtained between observed and calculated figure frequencies for an optimized fit. The computational burden, however, is immense, and the conclusions drawn from their analyses often differ little from the simpler, onevariable models (Chapman \& Kimber, 1992a and b).

There are occasions, however, in which it may be thought, a priori, that a single independent variable does not adequately represent the relationships amongst the genomes in a hybrid. This paper describes some such circumstances in tetraploid and pentaploid hybrids and presents pairing models, described as second-order models, incorporating two independent variables to better represent these situations. The terms pairing, meiotic figure, meiotic configuration, arm configuration, dataset, meiotic analysis and $c$ are used as discussed by Chapman \& Kimber (1992a).

\section{Theory}

There are three basic models for genome relationships in tetraploid hybrids. These are the $2: 2,2: 1: 1$ and $3: 1$ models exemplified by the genomic formulae AABB, $\mathrm{AABC}$ and $\mathrm{AAAB}$, respectively (Kimber \& Alonso, 1981; Chapman \& Kimber, 1992b). It is possible to envision an intermediate situation between the $2: 2$ and 2:1:1 models, which might be represented by the genomic formulae $\mathrm{AABB}^{\prime}$ where the second pair of genomes, $\mathrm{BB}^{\prime}$ has a lower affinity than the AA pair, but which is still appreciably higher than the affinity between any of the A and B genomes. This situation, which will be termed the $2:(1: 1)$ model, could arise when a tetraploid species is crossed with a synthetic amphiploid containing the two 'ancestral' genomes. It might also rise when two 'sibling' species are crossed which share a pivotal genome, but where a common, original, differential genome has been restructured in different ways (see Zohary \& Feldman 1962).

A second possibility arises when an allotetraploid species is crossed with a synthetic autotetraploid containing one of the same genomes. In these circum- 
stances the genomes from the autotetraploid would be expected to be similar to each other than they are to that from the allotetraploid. Such a situation, which would be intermediate between the $3: 1$ and $2: 1: 1$ models, can be described by the genomic formula $\mathrm{AAA}^{\prime} \mathrm{B}$ and represented as a $(2: 1): 1$ model, with both the $\mathrm{A}$ and $\mathrm{A}^{\prime}$ genomes considered equally dissimilar to the $\mathrm{B}$.

Analogous situations can exist at the pentaploid level as derivations of the 'first-order' models described by Espinasse \& Kimber (1981), and Chapman \& Kimber (1990c). One would be a $2:(1: 1) 1$ model with the genomes $\mathrm{AABB}^{\prime} \mathrm{C}$, intermediate between the $2: 2: 1$ and $2: 1: 1: 1$ models; another the $(2: 1): 1: 1$ model with the genomes $\mathrm{AAA}^{\prime} \mathrm{BC}$, intermediate between the $3: 1: 1$ and $2: 1: 1: 1$ models. A range of other intermediates are conceivable but it is thought that these are the ones most likely to be encountered in practice.

As with the first-order models, the relative affinity between the most similar genomes is denoted as $x$ and that between the least similar as $y$, where $x+y=1$ and $x \geq y$. The affinity between the genomes with the intermediate similarity is defined as $z$ where $x \geq z \geq y$. This relationship has been chosen so that when $z=x$ or $z=y$ it follows that one of the simpler, first-order models represents the best solution. The ratio of these affinities is expressed in the ratio of pairing at metaphase $I$ among the several pairwise combinations of genomes.

The relative affinities between the various possible pairs of genomes for both tetraploids and pentaploids are set out in Table 1. The ratio of these affinities is expressed in the ratio of pairing at metaphase I among the several pairwise combinations of genomes. Calculations for given values of $c$ (mean arm pairing frequency), $x$ and $z$ proceed in a similar manner to the first-order models (Chapman \& Kimber, 1992b and c), but incorporate $z$ rather than $x$ or $y$ as the value of relative affinity between the appropriate pairs of genomes. First the arm configuration frequencies (pairing behaviour of one set of homoeologous chromosome arms) are determined, and from these the resulting meiotic configurations and figure frequencies.

As with the first-order models, optimization commences with the calculation of $c$ from the observed numbers of figures. Then, as two variables, $x$ and $z$, are to be estimated, a two-dimensional search is initiated for the combination of values which minimizes the weighted sums of squares of differences (WSSD) between the observed and calculated figure numbers, subject to the constraints that $1 \geq x \geq 0.5$ and $x \geq z \geq y$. The computer programs previously described for firstorder models have been adapted for these analyses, first by inclusion of the relevant affinities and formulae, secondly by incorporating a routine that performs the
Table 1 Affinities between arm pairs for second-order models in tetraploid and pentaploid hybrids

\begin{tabular}{|c|c|c|c|c|c|}
\hline \multirow[b]{2}{*}{ Arm pair } & \multicolumn{5}{|c|}{ Model } \\
\hline & $2: 2$ & $2:(1: 1)$ & $2: 1: 1$ & $(2: 1): 1$ & $3: 1$ \\
\hline \multicolumn{6}{|c|}{ Tetraploids } \\
\hline $1-2$ & $x$ & $x$ & $x$ & $x$ & $x$ \\
\hline $1-3$ & $y$ & $y$ & $y$ & $z$ & $x$ \\
\hline $1-4$ & $y$ & $y$ & $y$ & $y$ & $y$ \\
\hline $2-3$ & $y$ & $y$ & $y$ & $z$ & $x$ \\
\hline $2-4$ & $y$ & $y$ & $y$ & $y$ & $y$ \\
\hline $3-4$ & $x$ & $z$ & $y$ & $y$ & $y$ \\
\hline \multirow{2}{*}{\multicolumn{2}{|c|}{$\begin{array}{l}\text { If } z=x, \text { then model }= \\
\text { If } z=y, \text { then model }=\end{array}$}} & \multirow{2}{*}{\multicolumn{2}{|c|}{$\begin{array}{l}2: 2 \\
2: 1: 1\end{array}$}} & \multirow{2}{*}{\multicolumn{2}{|c|}{$\begin{array}{l}3: 1 \\
2: 1: 1\end{array}$}} \\
\hline & & & & & \\
\hline & \multicolumn{5}{|c|}{ Model } \\
\hline & $2: 2: 1$ & $2:(1: 1): 1$ & $2: 1: 1: 1$ & $(2: 1): 1: 1$ & $3: 1: 1$ \\
\hline \multicolumn{6}{|c|}{ Pentaploids } \\
\hline $1-2$ & $x$ & $x$ & $x$ & $x$ & $x$ \\
\hline $1-3$ & $y$ & $y$ & $y$ & $z$ & $x$ \\
\hline $1-4$ & $y$ & $y$ & $y$ & $y$ & $y$ \\
\hline $1-5$ & $y$ & $y$ & $y$ & $y$ & $y$ \\
\hline $2-3$ & $y$ & $y$ & $y$ & $z$ & $x$ \\
\hline $2-4$ & $y$ & $y$ & $y$ & $y$ & $y$ \\
\hline $2-5$ & $y$ & $y$ & $y$ & $y$ & $y$ \\
\hline $3-4$ & $x$ & $z$ & $y$ & $y$ & $y$ \\
\hline $3-5$ & $y$ & $y$ & $y$ & $y$ & $y$ \\
\hline $4-5$ & $y$ & $y$ & $y$ & $y$ & $y$ \\
\hline \multirow{2}{*}{\multicolumn{2}{|c|}{$\begin{array}{l}\text { If } z=x, \text { then model }= \\
\text { If } z=y, \text { then model }=\end{array}$}} & \multicolumn{2}{|l|}{$2: 2: 1$} & \multicolumn{2}{|l|}{$3: 1: 1$} \\
\hline & & \multicolumn{2}{|l|}{$2: 1: 1: 1$} & \multicolumn{2}{|l|}{$2: 1: 1: 1$} \\
\hline
\end{tabular}

optimization in two dimensions. These programs are written in TURBO PASCAL for the Apple Macintosh.

\section{Results and discussion}

Figures 1 and 2 illustrate the variation of calculated meiotic figure numbers with $z$ for notional tetraploid hybrids with a basic number of 7 where $c=0.5$ and $x=0.95$, all values representative of those found in analyses of real datasets. As $z$ increases, so the models shift from $2: 1: 1$, through $2:(1: 1)$ or $(2: 1) 1$, to $2: 2$ or $3: 1$, respectively. For the $2:(1: 1)$ model the plots show that the estimated value of $z$ will depend largely on the frequencies of univalents, and rod and ring bivalents. For the $(2: 1): 1$ model univalents, ring bivalents and trivalents will be the more important figures. Quadrivalent numbers are low and relatively stable with respect to $z$. At higher values of $z$ the slopes for all figures diminish and it may be difficult to differentiate a $2:(1: 1)$ 
model where say $z>0.7$ from the $2: 2$ model, or a $(2: 1): 1$ model with $z>0.6$ from a $3: 1$ model. Similar observations and considerations apply to the $2:(1: 1:): 1$ and $(2: 1): 1: 1$ models for pentaploid hybrids.

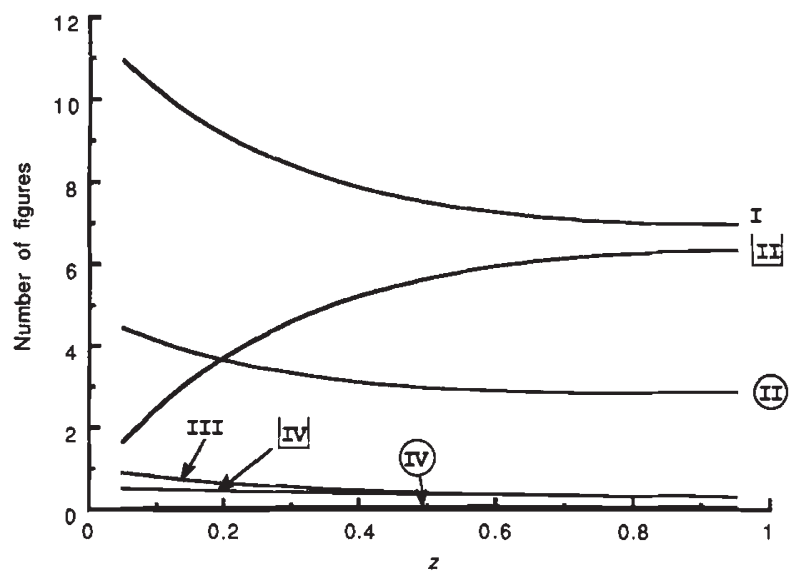

Fig. 1 The variation of meiotic figure numbers with $z$ for the $2:(1: 1)$ model. $(c=0.5, x=0.95$, and the basic chromosome number $=7)$.
Table 2 illustrates the application of the models described above. The first two entries are recently synthesized allotraploids in the genus Triticum and are representative of 12 datasets for amphidiploids listed

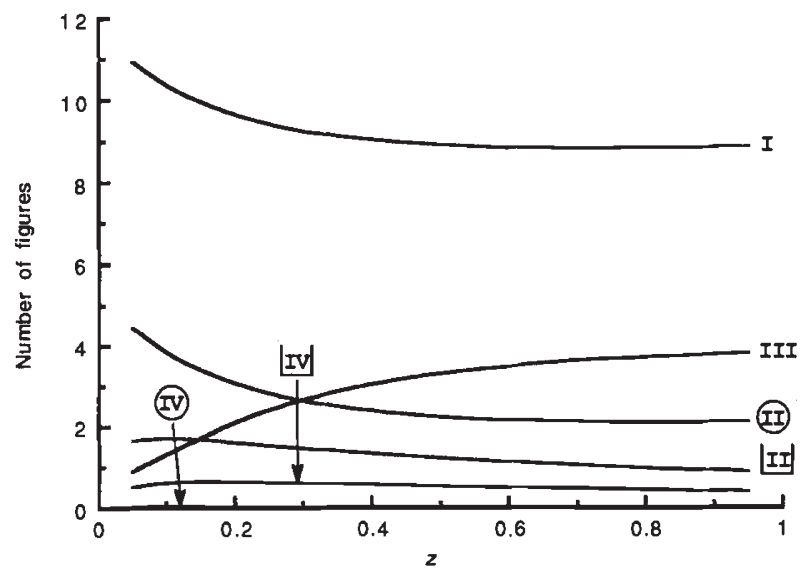

Fig. 2 The variation of meiotic figure numbers with $z$ for the (2:1): 1 model. ( $c=0.5, x=0.95$, and the basic chromosome number $=7$ ).

Table 2 The application of the second-order analyses to some tetraploid hybrids

\begin{tabular}{|c|c|c|c|c|c|c|c|c|c|c|c|}
\hline Hybrid and genomes & I & III & (II) & III & IV & (IV) & $c$ & Model & $x$ & $z$ & WSSD \\
\hline \multirow{4}{*}{$\begin{array}{l}\text { 1. Triticum monococcum } \times \\
\text { T. tauschii amph. AADD }\end{array}$} & 0.00 & 2.79 & 11.20 & 0.00 & 0.00 & 0.00 & 0.900 & & & & \\
\hline & 0.28 & 2.52 & 11.28 & 0.00 & 0.01 & 0.02 & & $2: 2$ & 0.999 & \multirow{3}{*}{0.998} & 0.409 \\
\hline & 0.28 & 2.51 & 11.26 & 0.00 & 0.01 & 0.02 & & $2:(1: 1)$ & 0.998 & & 0.417 \\
\hline & 0.32 & 0.92 & 3.81 & 0.16 & 1.41 & 3.03 & & $2: 1: 1$ & 0.556 & & 411.164 \\
\hline \multirow{4}{*}{$\begin{array}{l}\text { 2. T. longissimum } \times \\
\text { T. umbellualtum amph. } \\
\text { S'S'UU }\end{array}$} & 0.67 & 3.21 & 10.13 & 0.10 & 0.10 & 0.00 & 0.856 & & & \multirow{4}{*}{0.833} & \\
\hline & 0.58 & 3.40 & 10.09 & 0.01 & 0.04 & 0.06 & & $2: 2$ & 0.996 & & 0.354 \\
\hline & 0.74 & 3.23 & 10.21 & 0.01 & 0.06 & 0.08 & & $2:(1: 1)$ & 0.995 & & 0.214 \\
\hline & 0.66 & 1.29 & 3.49 & 0.32 & 1.76 & 2.45 & & $2: 1: 1$ & 0.584 & & 331.566 \\
\hline \multirow{4}{*}{$\begin{array}{l}\text { 3. }(T . \text { monococcum } \times \text { T. tauschii) } \times \\
(T . \text { monococcum } \times \text { T. uniaristatum }) \\
\text { AADN }\end{array}$} & 6.25 & 3.93 & 4.25 & 1.50 & 0.13 & 0.00 & 0.565 & & & \multirow{4}{*}{0.298} & \\
\hline & 5.30 & 5.69 & 3.10 & 0.52 & 0.68 & 0.22 & & $2: 2$ & 0.903 & & 32.752 \\
\hline & 7.05 & 3.93 & 4.16 & 0.60 & 0.61 & 0.14 & & $2:(1: 1)$ & 0.945 & & 11.895 \\
\hline & 7.31 & 2.25 & 3.16 & 1.41 & 1.21 & 0.20 & & $2: 1: 1$ & 0.865 & & 36.647 \\
\hline \multirow{4}{*}{ 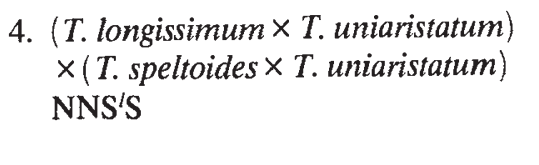 } & 6.54 & 4.74 & 4.39 & 0.94 & 0.10 & 0.00 & 0.561 & & & \multirow{4}{*}{0.374} & \\
\hline & 5.40 & 6.09 & 3.48 & 0.36 & 0.45 & 0.14 & & $2: 2$ & 0.939 & & 17.277 \\
\hline & 6.82 & 4.73 & 4.34 & 0.37 & 0.39 & 0.10 & & $2:(1: 1)$ & .965 & & 4.508 \\
\hline & 7.15 & 2.51 & 2.92 & 1.41 & 1.23 & 0.21 & & $2: 1: 1$ & 0.853 & & 51.974 \\
\hline \multirow{4}{*}{$\begin{array}{l}\text { 5. Hordeum brachyantherum } \times \\
\text { H. fuegianum }\end{array}$} & 8.90 & 5.40 & 3.80 & 0.25 & 0.00 & 0.00 & 0.482 & & & \multirow{4}{*}{0.351} & \\
\hline & 7.51 & 6.60 & 2.88 & 0.20 & 0.19 & 0.04 & & $2: 2$ & 0.969 & & 11.723 \\
\hline & 8.90 & 5.39 & 3.77 & 0.12 & 0.09 & 0.02 & & $2:(1: 1)$ & 0.990 & & 0.295 \\
\hline & 9.47 & 3.05 & 2.72 & 1.23 & 0.74 & 0.08 & & $2: 1: 1$ & 0.893 & & 44.664 \\
\hline \multirow{4}{*}{$\begin{array}{l}\text { 6. T. columnare } \times 4 \times \text { T. umbellulatum } \\
\text { UUUM }\end{array}$} & 8.00 & 2.05 & 2.35 & 2.65 & 0.70 & 0.05 & 0.512 & & & \multirow{4}{*}{0.402} & \\
\hline & 8.51 & 2.88 & 2.77 & 1.33 & 0.93 & 0.12 & & $2: 1: 1$ & 0.876 & & 20.283 \\
\hline & 8.33 & 1.86 & 2.23 & 2.61 & 0.85 & 0.06 & & $(2: 1): 1$ & 0.914 & & 0.703 \\
\hline & 7.95 & 1.99 & 1.87 & 2.81 & 0.90 & 0.07 & & $3: 1$ & 0.863 & & 1.825 \\
\hline \multirow{4}{*}{$\begin{array}{l}\text { 7. T. columnare } \times 4 \times T \text {. umbellulatum } \\
\text { UUUM }\end{array}$} & 8.70 & 2.85 & 3.60 & 1.60 & 0.20 & 0.20 & 0.523 & & & \multirow{4}{*}{0.098} & \\
\hline & 8.94 & 2.27 & 3.43 & 1.27 & 0.87 & 0.09 & & $2: 1: 1$ & 0.903 & & 9.888 \\
\hline & 8.93 & 2.27 & 3.42 & 1.27 & 0.87 & 0.10 & & $(2: 1): 1$ & 0.902 & & 9.889 \\
\hline & 7.30 & 2.64 & 1.74 & 2.28 & 1.13 & 0.14 & & $3: 1$ & 0.802 & & 34.073 \\
\hline
\end{tabular}

Sources of data: 1-4, Dajun \& Kimber (1982); 5, Bothmer et al. (1988); 6-7, Kimber \& Yen (1989). 
by Dajun \& Kimber (1982). The first, a Triticum monococcum $\times T$. tauschii amphiploid, shows a clear fit with the 2:2 model with the optimized value of $z$ equal to that of $x$, and very close to one, when analysed for the $2:(1: 1)$ model. The second example, a $T$. longissimum $\times T$. umbellulatum amphiploid, shows a value of $z$ that differs somewhat from $x$. The fit, however, is scarcely better than that of the $2: 2$ model and, on the principle of parsimony, this is the prefered interpretation. Indeed, the simpler first-order models are to be preferred throughout if $z$ does not differ appreciably from $x$ or $y$, or if the WSSD for the second-order model does not differ appreciably from the WSSD for one of the relevant first-order models.

The next two examples are of the hybrids between such newly synthesized amphiploids. Example 3 with the genomes AADN would be expected to conform to a 2:1:1 model, with pairing confined to the two A genomes, which have their origin in T. monococcum. In fact the $2: 2$ model is a slightly better fit than the $2: 1: 1$, but that of the $2(1: 1)$ model is a substantial improvement. It appears that whilst most pairing is between the A genomes, the $\mathrm{D}$ and $\mathrm{N}$ genomes are also pairing somewhat, a little with the A genomes $(y=0.055)$ but largely between themselves. This may be interpreted in two ways. One option is that the $\mathrm{D}$ and $\mathrm{N}$ genomes are closer to each other than they are to the A genomes. The alternative is that because the A genomes are truly homologous their own pairing competitively excludes associations with the $\mathrm{D}$ and $\mathrm{N}$ genomes, although $\mathrm{A}, \mathrm{D}$ and $\mathrm{N}$ may be equally dissimilar.

In the fourth example it is clear again that the $2:(1: 1)$ model is to be preferred. In this case, however, it is already known that the $S^{l}$ genome of $T$. longissimum is similar to the $\mathrm{S}$ of $T$. speltoides, both species being placed in the section Sitopsis (Kihara \& Tanaka, 1970). The values of $x$ and $z$ estimated here would indicate that there is about three times as much pairing between the $\mathbf{N}$ genomes as between the $\mathbf{S}$ and $\mathbf{S}^{l}$. This is in agreement with the conclusion of Yen \& Kimber (1989) that the $S$ and $S^{l}$ genomes are comparatively distinct.

The fifth example is a hybrid between two Hordeum species thought to have homologous or homoeologous genomes (Bothmer et al., 1988). The present analysis shows a good fit with a $2:(1: 1)$ model with a $y$ approaching zero, which suggests that the four genomes are pairing two by two, but that one pair, being more differentiated, does so only a third as frequently as the other.

Hybrids that involve autotetraploids are less common in the literature. The two examples shown in Table 2 both involve autotetraploid T. umbellulatum and T. columnare and come from Kimber \& Yen (1989). The first, based on 14 plants, fits the 3:1 model fairly well but the $(2: 1): 1$ model rather better. The second example, based on two plants, fits the 2:1:1 model. Kimber \& Yen (1989) concluded that while some accessions of $T$. columnare carried modified U genomes, in most cases they were little altered. The present analysis suggests rather that the $\mathrm{U}$ genome in $T$. columnare is modified in all accessions but to varying degrees.

The greater subtlety of these analyses has been used to examine one particular question in the genus Triticum, the relationship between the $B$ and $G$ genomes. Some nine hybrids between T. turgidum and T. timopheevii and 11 between T. aestivum and T.

Table 3 The meiotic analysis of hybrids between T. turgidum and T. timopheevii

\begin{tabular}{|c|c|c|c|c|c|c|c|c|}
\hline & \multirow[b]{3}{*}{$c$} & \multicolumn{7}{|l|}{ Model } \\
\hline & & \multicolumn{2}{|l|}{$2: 1: 1$} & \multicolumn{3}{|l|}{$2:(1: 1)$} & \multicolumn{2}{|l|}{$2: 2$} \\
\hline & & $x$ & WSSD & $x$ & $z$ & WSSD & $x$ & WSSD \\
\hline 1. & 0.509 & 0.827 & 62.931 & 0.988 & 0.479 & 0.086 & 0.975 & 4.030 \\
\hline 2. & 0.477 & 0.861 & 29.622 & 0.962 & 0.368 & 3.550 & 0.931 & 10.152 \\
\hline 3. & 0.525 & 0.869 & 19.219 & 0.938 & 0.274 & 4.042 & 0.882 & 21.325 \\
\hline 4. & 0.572 & 0.858 & 53.644 & 0.964 & 0.357 & 7.184 & 0.936 & 23.189 \\
\hline 5. & 0.605 & 0.790 & 95.218 & 0.978 & 0.511 & 4.280 & 0.957 & 9.358 \\
\hline 6. & 0.594 & 0.832 & 85.517 & 0.978 & 0.440 & 3.502 & 0.963 & 13.525 \\
\hline 7. & 0.657 & 0.761 & 123.317 & 0.978 & 0.516 & 8.026 & 0.968 & 14.421 \\
\hline 8. & 0.594 & 0.500 & 99.845 & 0.983 & 0.596 & 1.962 & 0.976 & 3.774 \\
\hline 9. & 0.598 & 0.759 & 101.845 & 0.984 & 0.545 & 1.404 & 0.976 & 4.977 \\
\hline
\end{tabular}

Sources of data: 1-2, Shands \& Kimber (1973); 3, Kimber \& Alonso (1981); 4-7 Bell \& Sachs (1953); 8-9 Kushnir \& Halloran (1983). 
Table 4 The meiotic analysis of hybrids between T. aestivum and T. timopheevii

\begin{tabular}{|c|c|c|c|c|c|c|c|c|}
\hline & \multirow[b]{3}{*}{$c$} & \multicolumn{7}{|c|}{ Model } \\
\hline & & \multicolumn{2}{|c|}{$2: 1: 1: 1$} & \multicolumn{3}{|c|}{$2:(1: 1): 1$} & \multicolumn{2}{|l|}{$2: 2: 1$} \\
\hline & & $x$ & WSSD & $x$ & $z$ & WSSD & $x$ & WSSD \\
\hline 1. & 0.366 & 0.941 & 2.109 & 0.960 & 0.169 & 0.791 & 0.930 & 8.731 \\
\hline 2. & 0.570 & 0.901 & 29.594 & 0.961 & 0.418 & 3.824 & 0.950 & 9.489 \\
\hline 3. & 0.639 & 0.937 & 35.653 & 0.975 & 0.264 & 3.878 & 0.971 & 53.643 \\
\hline 4. & 0.545 & 0.914 & 22.162 & 0.963 & 0.348 & 3.202 & 0.950 & 12.835 \\
\hline 5. & 0.487 & 0.924 & 9.564 & 0.960 & 0.270 & 1.228 & 0.942 & 12.477 \\
\hline 6. & 0.578 & 0.868 & 43.751 & 0.958 & 0.762 & 2.575 & 0.954 & 2.622 \\
\hline 7. & 0.507 & 0.926 & 15.480 & 0.969 & 0.303 & 1.014 & 0.956 & 13.140 \\
\hline 8. & 0.604 & 0.867 & 46.135 & 0.955 & 0.696 & 4.001 & 0.949 & 4.188 \\
\hline 9. & 0.598 & 0.944 & 18.076 & 0.970 & 0.192 & 5.065 & 0.959 & 65.934 \\
\hline 10. & 0.501 & 0.938 & 8.906 & 0.960 & 0.181 & 4.272 & 0.937 & 30.438 \\
\hline 11. & 0.671 & 0.904 & 52.809 & 0.964 & 0.395 & 8.761 & 0.955 & 23.294 \\
\hline
\end{tabular}

Sources of data: 1-2, Dhaliwal (1977); 3, Watanabe (1953); 4-9 Watanabe \&

Mukade (1955); 10-11, Leont'ev \& Budashkina(1981).

timopheevii have been identified where the meiotic data were detailed enough for analyses to be carried out (Tables 3 and 4). All the hybrids between $T$. turgidum $\times$ T. timopheevii showed a better fit with the $2:(1: 1)$ model than either the $2: 1: 1$ or $2: 2$ models, with $x$ varying between 0.938 and 0.988 , and $z$ between 0.274 and 0.596 . In contrast, whilst the $T$. aestivum $\times$ T. timopheevii hybrids show similar values of $x$, varying from 0.955 to 0.975 , those of $z$ vary more widely, from 0.169 to 0.762 for the $2:(1: 1): 1$ model. Two examples ( 6 and 8 ) are essentially $2: 2: 1$ solutions, for the WSSD for the $2:(1: 1): 1$ model is only slightly smaller than that of the 2:2:1 model. Despite this the value of $z$ for the pentaploids is, on average, somewhat lower than that for the tetraploids.

Given that the $x$ value reflects the relative affinity of the A genomes, and $z$ that of the B and G genomes, these analyses defy simple explanation. The similarity of the $z$ values within the tetraploid hybrids indicates that the $B$ and $G$ genomes sampled are uniform within species and differ comparably between $T$. turgidum and T. timopheevii. The lower average value of $z$ in the pentaploid hybrids might be attributed to the action of the Ph2 locus on chromosome 3D, which inhibits pairing between homoeologous genomes (Mello-Sampayo, 1971) but it does not explain the greater range of $z$. Presumably either or both of the B or G genomes sampled in this range of hybrids show greater variability than those sampled in the tetraploid hybrids.

Equivocal though these analyses are, they do highlight the hazard of basing genome analysis on one or a few interspecific hybrid plants. As with other measures of genetic distance, variation within as well as between species should be anticipated. Thus a range of hybrids based on several accessions of each species is highly desirable, although in practice they may be difficult to obtain. Similarly, merging datasets together, prior to analysis, is likely to give a simplistic image of genome relationships. Analysing each separately may reveal a more complex, but realistic, picture.

The second-order models developed here represent the practical limit for meiotic analysis based on the cytogenetics of simple interspecific hybrids. Although there are six or seven possible figure types observed in tetraploid and pentaploid meioses, it is often the case that quadrivalents and quinquevalents are infrequent due to low levels of pairing, and can provide little information. Additional parameters to $c, x, y$ and $z$ could be postulated and better-fit solutions found, but the computational burden would greatly increase without much improvement in understanding the genomic relationships involved.

Further advancement depends rather on more elaborate techniques enabling the particular genomes and chromosomes involved in the meiotic figures to be directly identified, e.g. through the use of telocentric chromosomes or banding patterns (see Alonso \& Kimber, 1983; or Cuñado et al., 1986; for examples of their use). The simplicity of the practical work involved in recording meiotic figures and the increasing availability of computers to analyse the data, ensure a continuing role for classic meiotic studies in species hybrids as a valuable technique in its own right, or as a preliminary to more costly investigations. 


\section{A note on programs}

All the programs for the analysis of meiosis in species hybrids described in this series of papers have been written in TURBO PASCAL to run on the Apple Macintosh. Copies of the source code and the compiled programs are available from the authors upon receipt of an $800 \mathrm{~K}$ or $1.4 \mathrm{MB}$ initialized disk. Use of the source code requires TURBO PASCAL.

\section{Acknowledgements}

CGDC was supported by a post-doctoral fellowship from the University of Missouri. Contribution from the Missouri Agricultural Experiment Station. Journal Series Number: 11,261.

\section{References}

ALONSO, L. C. AND KIMBER, G. 1983. A study of genome relationships in wheat based on telocentric chromosome pairing. II. Z. Pflanzenzüchtg., 90, 273-284.

BELL, G. D. H. AND SACHS, L. 1953. Investigations in the Triticinae II. The cytology and fertility of intergeneric and interspecific $\mathrm{F}_{1}$ hybrids and their derived amphidiploids. $J$. Agric. Sci., 43, 105-115.

BOTHMER, R., VON, FL.INK, J. AND LANDSTRÖM, T. 1988. Meiosis in interspecific Hordeum hybrids. IV. Tetraploid $(4 x \times 4 x)$ hybrids. Genome, 30, 479-485.

CHAPMAN, C. G. D. AND KIMBER, G. 1992a. Developments in the meiotic analysis of hybrids. I. Review of theory and optimization in triploids. Heredity, 68, 97-103.

CHAPMAN, C. G. D. AND KIMBER, G. 1992b. Developments in the meiotic analysis of hybrids. II. Amended models for tetraploids. Heredity, 68, 105-113.

CHAPMAN, C. G. D. AND KIMBER, G. 1992c. Developments in the meiotic analysis of hybrids. III. Amended models for pentaploids. Heredity, 68, 193-200.

CHAPMAN, C. G. D. AND KIMBER, G. 1992d. Developments in the meiotic analysis of hybrids. IV. Utilizing datasets with merged classes. Heredity, 68, 201-204.

CRANE, C. F. AND SLEPER, D. A. 1989a. A model of meiotic chromosome association in triploids. Genome, 32, 82-98.

CRANE, C. F. AND SLEPER, D. A. 1989b. A model of meiotic chromosome association in tetraploids. Genome, 32 , 691-707.

CUÑADO, N., CERMEÑO, M. C. AND ORELLANA, J. 1986. Interactions between wheat, rye and Aegilops ventricosa chromosomes on homologous and homoeologous pairing. Heredity, 56, 219-226.

CUÑADO, N., CERMEÑO, M. C. AND ORELLANA, J. 1988. Interactions between wheat, rye and Aegilops ventricosa chromosomes on homologous and homoeologous pairing. Heredity, 56, 219-226.

DAJUN, L. AND KIMBER, G. 1982. The utilization of $4 x$ amphidiploids in the genomic analysis of wheat. $Z$. Pflanzenzüchtg., 88, 302-310.

DHALIWAL, H. S. 1977 . The $P h$ gene and the origin of tetraploid wheats. Genetica, 47, 177-182.

ESPINASSE, A. AND KIMBER, G. 1981. The analysis of meiosis in hybrids. IV Pentaploid hybrids. Can. J. Genet. Cytol. 23, 235-254.

KIHARA, H. AND TANAKA, M. 1970. Addendum to the classification of the genus Aegilops by means of genome analysis. Wheat Information Service, 30, 1-2.

KIMBER, G. AND ALONSO, L. C. 1981. The analysis of meiosis in hybrids. III. Tetraploid hybrids. Can. J. Genet. Cytol., 23, 235-254.

KIMBER, G. AND YEN, Y. 1989. Hybrids involving wheat relatives and autotetraploid Triticum umbellulatum. Genome, 32 , 1-5.

KUSHNIR, U. AND HALLORAN, G. M. 1983. Evidence on the origin of the $G$ genome in wheat: cytology and fertility of a Triticum timopheevi-like mutant. Can. J. Genet. Cytol., 25, 651-661.

LEONT'EV, F. P. AND BUDASHKINA, E. B. 1981. Cytological study of interspecific pentaploid Triticum aestivum $\times T$. timopheevii $\mathrm{F}_{1}$ hybrids. Cytol. Genet. 14, 54.

MELlO-SAMPAYO, M. 1971. Genetic regulation of meiotic chromosome pairing by chromosome 3D of Triticum aestivum. Nat. New Biol., 230, 22-23.

SHANDS, H. AND KIMBER, G. 1973. Reallocation of the genomes of Triticum timopheevii Zhuk. Proc. Fourth Int. Wheat Gen. Symp., 101-108.

WATANABE, Y. 1953. Studies on meiosis, morphology and fertility in $\mathrm{F}_{1}$ hybrids: Triticum timopheevii Zhuk. $\times$ Triticum vulgare. Jap. J. Breeding, 2, 173-177.

WATANABE, Y. AND MUKADE, $\mathrm{K}, 1955$. Maturation division if $F_{1}$ hybrids between five species in the dinkel group and Triticum timopheevii Zhuk. Jap. J. Genet., 30, 24-33.

YEN, Y. AND KIMBER, G. 1989. A triploid hybrid between autotetraploid Triticum longissimum and T. speltoides. Cereal Res. Commum., 17, 259-264.

ZOHARY, D. AND FELDMAN, M. 1962. Hybridization between amphidiploids and the evolution of polyploids in the wheat (Aegilops-Triticum) group. Evolution, 16, 44-61. 